\title{
Effet de quelques facteurs intrinsèques ou extrinsèques d'affaiblissement des tournesols sur leur sensibilité au dessèchement précoce
}

\author{
Pierre DAVET, Michel HERBACH, Michel RABAT \& Georges PIQUEMAL $\left({ }^{*}\right)$ \\ I.N.R.A., Laboratoire de Biologie et Pathologie végétales de l'E.N.S.A., place Viala, Centre de Recherches de \\ Montpellier, F 34060 Montpellier Cedex \\ (*) I.N.R.A., Station d'Amélioration des Plantes, Domaine de Melgueil, Centre de Recherches de Montpellier, \\ F 34130 Mauguio
}

Mots clés additionnels : Macrophomina phaseolina, Fusarium spp., Cylindrocarpon spp., Helianthus annuus, racines, tiges, température, stress, sécheresse, comportement variétal.

Additional key words : Macrophomina phaseolina, Fusarium spp., Cylindrocarpon spp., Helianthus annuus, roots, stems, temperature, stress, drought, selection.

\section{INTRODUCTION}

Des symptômes de dessèchement précoce des tiges de tournesol peuvent être fréquemment observés dans les cultures du Sud et du Centre de la France, et l'on peut généralement les associer à la présence d'un champignon du sol, Macrophomina phaseolina (Tassi) Goid. Ce champignon, d'origine tropicale, a une aire de répartition très large et une gamme d'hôtes très étendue (DHingRa \& Sinclair, 1978). En Europe, il a été signalé sur le tournesol en Yougoslavie par ACIMOVIC (1962), en France par AlabouvetTe \& Bremeersch
(1975), en Espagne par SACKSTON \& JiMENEZ-DiAZ (1976) et au Portugal par BARros (1980). Tous ces auteurs considèrent que $M$. phaseolina peut être responsable de pertes de rendement importantes en cultures non irriguées.

Cependant, en France, dans les tiges des plantes malades, nous isolons régulièrement divers Fusarium (en particulier F. solani (Mart.) (App. et Wr.) Sn. et H.), des Cylindrocarpon spp. ou des Alternaria spp. en même temps que $M$. phaseolina et parfois même en son absence. D'autre part, les tournesols sont très peu, ou pas du tout, colonisés pendant la période de 
leur développement végétatif. Par contre, au début de la floraison, soit 80 à $90 \mathrm{j}$ au moins après le semis, le système racinaire est progressivement envahi. Les champignons gagnent ensuite la tige et se répandent dans la moelle en quelques jours (SAUMON et al., 1984). Il semblerait donc que, tout comme c'est le cas pour la pourriture des tiges du maïs (BARRIÈRE et al., 1981), divers micromycètes puissent envahir les tiges du tournesol à partir d'un certain moment, apparemment déterminé par l'état physiologique de la plante.

Nous nous proposons, dans ce mémoire, d'étudier quelques facteurs susceptibles de modifier l'état physiologique des tournesols et de favoriser leur envahissement. Nous nous intéresserons plus particulièrement à $M$. phaseolina puisque ce champignon est le plus régulièrement mis en évidence.

\section{MATÉRIEL ET MÉTHODES}

\section{A. Emplacement des essais}

Tous les essais de plein champ ont été mis en place dans le Sud-Ouest, à Loudes, près de Castelnaudary, sur les terrains de la Coopérative Agricole Lauragaise. Le sol, argilo-calcaire, contient 3,3 p. 100 de calcaire total et 1,7 p. 100 de matière organique. Son $\mathrm{pH}$ est de 7,6 (eau) ou 6,8 (KCl). Toutes les parcelles contenaient un inoculum naturel de $M$. phaseolina, estimé en début de culture à 3 ou 4 propagules par g de sol, selon la technique de Mc CAIN \& SMITH (1972). Le précédent cultural était un maïs ou un blé.

\section{B. Données climatologiques}

Toutes les données climatologiques proviennent des relevés assurés par la Coopérative Agricole Lauragaise sur les lieux des expérimentations.

Le total des précipitations peut varier considérablement d'une année à l'autre : par exemple il est tombé $769 \mathrm{~mm}$ en 1982 et $466 \mathrm{~mm}$ en 1983. Les différences sont beaucoup moins marquées pour la période qui intéresse plus particulièrement la culture du tournesol. Ainsi, du mois de mars à la fin du mois d'août, il est tombé 308,281 et $306 \mathrm{~mm}$ respectivement en 1982, 1983 et 1984 (les E.T.P. correspondantes étant de 774, 721 et $746 \mathrm{~mm}$ ). Cependant, la répartition des pluies pendant ces 6 mois a été très différente en 1983 et en 1984 (fig. 1).

La température du sol, mesurée à 10 ou à $20 \mathrm{~cm}$ de profondeur, ne commence à dépasser $20^{\circ} \mathrm{C}$ que durant la $1^{\mathrm{re}} \mathrm{ou}$, parfois la $2^{\mathrm{e}}$ semaine du mois de juin. Juillet est le mois le plus chaud, avec des températures maximales de 26 à $29^{\circ} \mathrm{C}$ et des moyennes mensuelles de 22,5 à $25,5^{\circ} \mathrm{C}$, selon les années, à $20 \mathrm{~cm}$ de profondeur. Les températures maximales enregistrées dans le sol au mois d'août oscillent entre 23 et $28^{\circ} \mathrm{C}$. Les températures maximales relevées sous abri sont de 33 à $40^{\circ} \mathrm{C}$ en juillet et de 31 à $33^{\circ} \mathrm{C}$ en août.

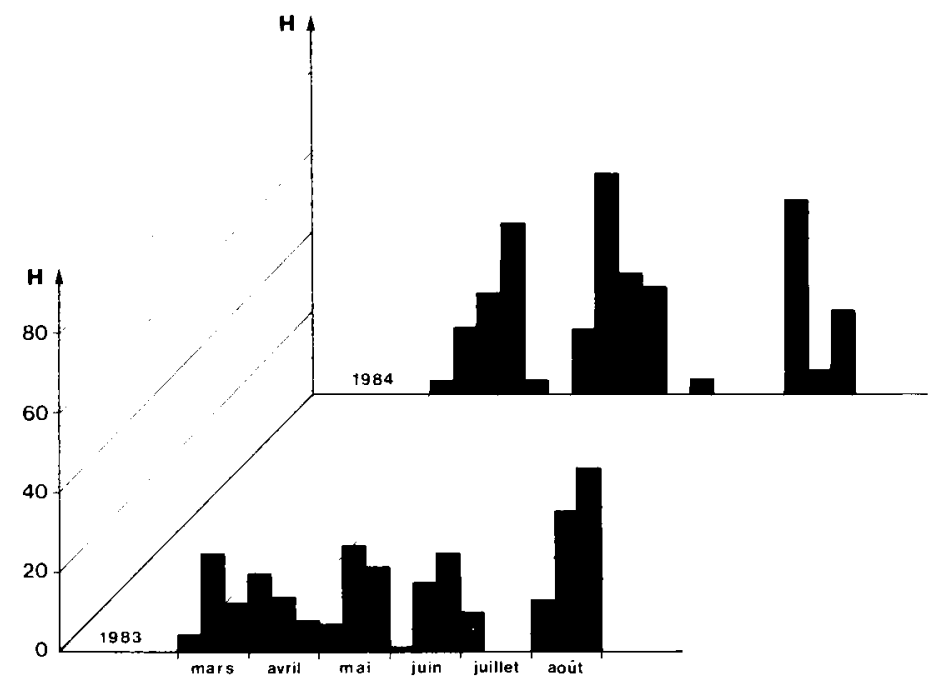

Figure 1

Relevés décadaires des chutes de pluie (exprimées en $\mathrm{mm}$ ) enregistrées à Loudes (Lauragais) en 1983 et en 1984.

Rainfall recorded during ten-day periods at Loudes (Lauragais) in 1983 and 1984 (expressed in $\mathrm{mm}$ ).

\section{Variétés de tournesol}

La variété semi-précoce «Mirasol 》 a été utilisée en 1982 pour le $1^{\text {er }}$ essai d'effeuillage. Les années suivantes, la variété semi-précoce "Rodéo " a été employée dans toutes les expérimentations.

Les essais de comportement variétal ont été réalisés avec des hybrides simples expérimentaux de la Station d'Amélioration des Plantes de l'I.N.R.A. (Montpellier).

\section{Semis}

Dans le cas des essais de comportement variétal, les semis ont été faits manuellement, en une ligne de 30 à 40 graines par variété. Les lignes étaient espacées de $60 \mathrm{~cm}$.

Pour les autres essais, les semis ont été exécutés mécaniquement, avec un espacement sur les lignes de $20 \mathrm{~cm}$ et des interlignes de $60 \mathrm{~cm}$. Pour étudier l'effet de la profondeur, on a comparé des semis réalisés le même jour, dans une parcelle horizontale homogène, à des profondeurs de $3-4 \mathrm{~cm}, 5-6 \mathrm{~cm}$ et $7-8 \mathrm{~cm}$, à raison de 8 lignes de $30 \mathrm{~m}$ de long pour chaque modalité. Dans tous les autres cas, la profondeur était réglée à $3 \mathrm{~cm}$.

Les semis ont toujours eu lieu entre le 8 et le 15 avril. Dans un cas particulier cependant, nous avons suivi l'évolution de la colonisation des racines de tournesols semés dans 2 parcelles contiguës, d'une part, le 16 avril, d'autre part, le 5 juin.

Toutes les cultures ont été conduites en sec. 


\section{E. Essais d'effeuillage et de décapitation des tourne- sols}

Dans l'essai mis en place en 1982, nous avons comparé les 3 traitements suivants :

- décapitation des plantes $99 \mathrm{j}$ après le semis, après l'apparition des boutons floraux et suppression, par la suite, des boutons axillaires éventuels ;

- enlèvement des feuilles du tiers inférieur des plantes $99 \mathrm{j}$ après le semis et suppression de quelques autres feuilles $18 \mathrm{j}$ plus tard, de façon à conserver les 6 feuilles bien développées supérieures;

- lot témoin non effeuillé ni décapité.

Les traitements ont été répétés en 4 blocs comprenant environ 60 plantes par parcelle élémentaire. Chaque dispositif était entouré de bordures non traitées.

Dans un autre essai, réalisé en 1983, on a comparé :

- enlèvement des feuilles du tiers supérieur des tournesols $77 \mathrm{j}$ après le semis,

- enlèvement des feuilles du tiers supérieur $107 \mathrm{j}$ après le semis,

- lot témoin non effeuillé.

les trailements élaient réparlis en 3 blocs.

\section{F. Essais d'indexation}

Dans une grande parcelle travaillée et semée de façon uniforme, nous avons repéré à l'aide d'une étiquette, un mois après la levée, environ 200 plantes ayant une taille réduite ou un diamètre inférieur à la moyenne et réparties dans l'ensemble du champ. A l'époque de la récolte, ces plantes ont été arrachées et comparées à une quantité équivalente de plantes voisines non étiquetées, considérées comme «normales ».

\section{G. Comportement vis-à-vis de la sécheresse}

Nous avons retenu 2 critères pour caractériser le comportement des tournesols vis-à-vis de la sécheresse : le rapporl ETR (évapotranspiration réelle)/ ETM (évapotranspiration maximum), calculé selon la méthode d'AlissA (1982) et la valeur de l'efficience de l'eau (BLANCHET et al., 1983).

Le principe de la $1^{\text {re }}$ mesure est le suivant : après germination dans l'eau, les graines sont repiquées sur de la pouzzolane et arrosées avec une solution minérale nutritive contenant, ou non, $89 \mathrm{~g} / \mathrm{l}$ de polyéthylèneglycol 6000 , dont le rôle est de diminuer la disponibilité de l'eau. Le rapport entre les quantités d'eau transpirées en présence d'eau pure et de polyéthylène-glycol (assurant ici une pression osmotique de 2 bars), mesurées pendant 4 semaines de culture en enceinte climatisée, traduit la capacité d'absorption des racines.

$L$ 'efficience de l'eau est évaluée par le rapport entre la quantité totale de matière sèche produite en 4 semaines et la quantité d'eau consommée pendant la même période.

\section{H. Notations}

L'état sanitaire et le degré de développement des tournesols ont été appréciés en cours et en fin de culture sur des échantillons de 10 à 15 plantes par parcelle élémentaire, arrachées avec précaution. On a compté le nombre de plantes sèches et, après avoir coupé les tiges en deux dans le sens longitudinal, le nombre de tiges contenant des sclérotes de $M$. phaseolina. On a mesuré aussi le diamètre des tiges au collet et la longueur de la portion de la moelle des tiges qui contenait des sclérotes. L'état des racines a été apprécié visuellement et noté de 0 (système radiculaire entièrement sain) à 5 (toutes les racines sont nécrosées). Six fragments de racine d'environ $4 \mathrm{~mm}$ ont été prélevés sur chaque plante, désinfectés superficiellement à l'hypochlorite de sodium, rincés et mis en culture sur le milieu $\mathrm{S}$ de MESSIAEN \& LAFON (1965) additionné d'acide citrique $(150 \mathrm{mg} / \mathrm{l})$ et de sulfate de streptomycine $(50 \mathrm{mg} / \mathrm{l})$. Les colonies ont été comptées après une semaine d'incubation à la lumière, à la température du laboratoire.

\section{RÉSULTATS}

\section{A. Colonisation des racines en fonction du temps et de la date de semis}

Les tournesols semés le 16 avril commencent à fleurir vers le 10 juillet et sont progressivement parasités après cette date, alors que ceux semés le 5 juin restent parfaitement sains pendant tout le mois de juillet et le début du mois d'août (fig. 2). Les plantes du $2^{\mathrm{e}}$ semis fleurissent à partir du 15 août et c'est seulement à ce moment que leurs racines sont colonisées par $M$. phaseolina.

La température du sol s'élève jusqu'à la fin du mois de juillet et commence à décroître au début du mois d'août.

\section{B. Effets de la suppression des capitules ou d'une partie du feuillage}

Les tournesols dont les capitules ont été supprimés ont des collets significativement plus gros que les autres et des racines beaucoup plus abondantes (tabl. 1). Toutes les plantes de ce traitement restent vertes jusqu'au mois de septembre ; leurs racines sont très peu nécrosées et aucun sclérote n'est visible dans la moelle.

Par contre, on observe des plantes sèches chez les témoins et dans les séries effeuillées dès la fin du mois d'août ; les racines sont moins abondantes et beaucoup sont nécrosées. Dans les 2 essais (1982 et 1983), le pourcentage de tiges envahies de microsclérotes est significativement plus élevé chez les plantes effeuillées que chez les témoins, mais la proportion de plantes effeuillées sèches est significativement différente des témoins seulement dans le $2^{e}$ essai. La note exprimant l'abondance des lésions sur les racines est toujours plus élevée chez les plantes effeuillées. 
TABLEAU

Effet des ablations foliaires ou florales sur l'envahissement des racines et des tiges par Macrophomina phaseolina.

Effect of leaf or capitulum removal on root and stem invasion by $\mathbf{M}$. phaseolina.

\begin{tabular}{|c|c|c|c|c|c|c|c|c|c|}
\hline \multirow[b]{2}{*}{ Traitements } & \multicolumn{5}{|c|}{ Essai de 1982} & ptembre & \multicolumn{3}{|c|}{$\begin{array}{l}\text { Essai de } 1983 \\
\text { Notation du } 25 \text { août }\end{array}$} \\
\hline & I & II & témoin & I & II & témoin & III & IV & témoin \\
\hline Diamètre du collet (mm) & $27,0_{\mathrm{a}}$ & $17,6_{b}$ & $20,0_{b}$ & $26,5_{a}$ & $15,5 \mathrm{c}$ & $18,1_{b}$ & $17,8_{\mathrm{b}}$ & $18,3_{b}$ & $19,9 \mathrm{a}$ \\
\hline Pourcentage de plantes sèches & $0_{\text {a }}$ & 20,8 & $12,5_{b}$ & 0 a & $58,3_{\mathrm{c}}$ & $12,5_{b}$ & $70,0_{\mathrm{b}}$ & $66,7_{b}$ & $20,0_{\mathrm{a}}$ \\
\hline Pourcentage de tiges envahies de microsclérotes & 0 a & 12,5 & $8,3_{b}$ & $0 \quad \mathrm{a}$ & $29,2 b$ & $\begin{array}{ll}0 & \\
& \end{array}$ & $96,7_{b}$ & $90,0_{b}$ & $53,3_{\mathrm{a}}$ \\
\hline Longueur des lésions de la moelle $(\mathrm{cm})$ & - & 一 & - & 一 & - & - & $40,4_{b}$ & $33,4_{b}$ & $13,7_{\mathrm{a}}$ \\
\hline Note d'état sanitaire des racines (de 0 à 5 ) & 0,9 & 2,2 & 1,2 & 1,5 & 4,2 & 2,1 & 4,5 & 4,1 & 2,1 \\
\hline Pourcentage de $M$. phaseolina isolé sur les racines & 13,2 & 6,2 & 9,0 & 20,1 & 16,7 & 10,5 & 64,0 & 50,7 & 52,0 \\
\hline
\end{tabular}

Traitement I : suppression des boutons floraux ; traitement II : ablation d'une partie du feuillage ; traitements III et IV : défoliation respectivement le 29 juin et le 29 juillet. Pour une même série de mesures, les chiffres suivis d'une même lettre ne sont pas différents au seuil de 5 p. 100 (tests de $t$ et de $\mathrm{X}^{2}$ ).

In 1982, cv. "Mirasol" was used in the experiments. In treatment I, every bud was cut off. In treatment II, the leaves from the lower third of the plants were suppressed on 16 July ; the remaining leaves, except the 6 well developed upper ones, were suppressed on 3 August. In 1983 , cv. "Rodéo" was used. In treatments III and IV, the leaves from the upper third of the plants were cut off on 29 June and 29 July, respectively. Root necrosis was assessed according to a scale from 0 (completely healthy roots) to 5 (roots entirely rotten). In each series of results, figures followed by the same letter were not significantly different at $5 \%$ level (Student's 1 or $\mathrm{X}^{2}$ test).
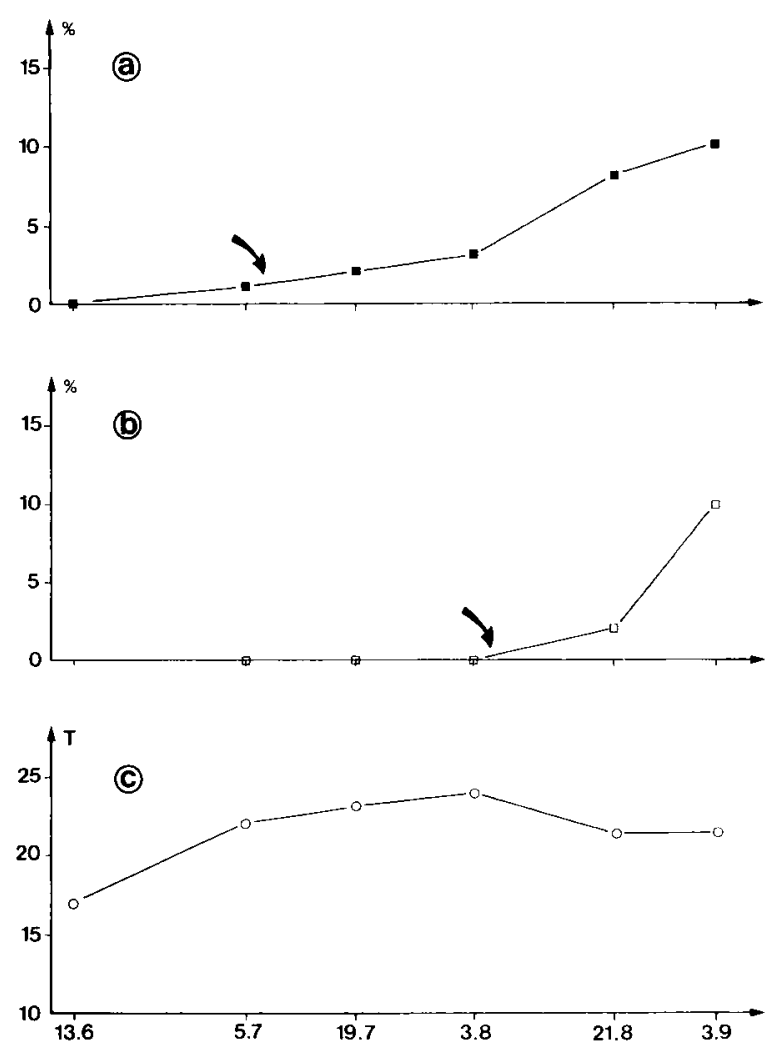

Figure 2

Colonisation par Macrophomina phaseolina des racines de tournesols semés à deux dates différentes : le 16 avril (a) et le 5 juin $(b)$, et évolution de la température du sol à $20 \mathrm{~cm}$ de profondeur (c). Les flèches indiquent le début de la floraison.

$\%$ of root pièces infected by $\mathrm{M}$. phaseolina in sunflowers seeded on two different dales: 16 April (a) and 5 June (b). and soil temperature (c) measured $20 \mathrm{~cm}$ below the surface leach point is the mean of the data for the seven days before the sampling date). The beginning of flowering is indicated by arrows.
L'ensemble des paramètres exprimant la vigueur des plantes et le degré d'attaque tend à indiquer que la suppression des étages supérieurs de feuilles à la fin du mois de juin affecte davantage les tournesols que la même opération réalisée fin juillet. Cependant, les résultats ne sont pas statistiquement différents au seuil de 5 p. 100.

Si l'on considère maintenant les colonies de $M$. phaseolina obtenues à partir des fragments de racines mis en culture, on constate que les pourcentages sont équivalents pour tous les traitements, dans les 2 essais.

\section{Effet de la sécheresse}

Dans un $1^{\text {er }}$ essai, 7 hybrides seulement sont comparés. On constate qu'il n'y a pas de sclérotes dans la moelle des tournesols dont le rapport ETR/ETM est élevé ; $M$. phaseolina est présent dans les tiges lorsque ETR/ETM est inférieur à 0,70 (tabl. 2).

L'essai mis en place l'année suivante porte sur 18 hybrides. L'analyse des résultats fait apparaître des corrélations de rang positives élevées entre les différents paramètres utilisés pour caractériser l'état des plantes en fin de culture (tabl. 3). Ce groupe de variables est corrélé négativement avec le rapport ETR/ETM. Il n'y a par contre aucune relation apparente entre la mesure de l'efficience de l'eau et l'un quelconque des autres paramètres. On note enfin une corrélation positive entre le rapport ETR/ETM et le nombre de jours compris entre la date du semis et celle de la floraison. 
TABLEAU 2

Relation entre la résistance à la sécheresse de quelques variétés et leur envahissement par M. phaseolina (résultats de 1982). Relation between drought tolerance and invasion by $\mathrm{M}$. phaseolina (1982 results).

\begin{tabular}{|c|c|c|c|c|c|}
\hline Variétés & $\frac{\mathrm{ETR}}{\mathrm{ETM}} \times 100$ & $\begin{array}{l}\text { Pourcentage } \\
\text { de plantes } \\
\text { sèches }\end{array}$ & $\begin{array}{l}\text { Pourcentage de } \\
\text { tiges contenant } \\
\text { des microsclérotes }\end{array}$ & $\begin{array}{l}\text { Note moyenne } \\
\text { d'état sanitaire } \\
\text { des racines }\end{array}$ & $\begin{array}{c}\text { Pourcentage } \\
\text { d'isolements } \\
\text { de } M \text {. phaseolina } \\
\text { sur les racines }\end{array}$ \\
\hline 1 & 96,6 & 0 & 0 & 1,5 & 0 \\
\hline 2 & 86,8 & 16,7 & 0 & 1,8 & 16,7 \\
\hline 3 & 86,8 & 0 & 0 & 3,0 & 10,0 \\
\hline 4 & 73,9 & 0 & 0 & 2,4 & 10,0 \\
\hline 5 & 72,6 & 0 & 0 & 1,2 & 6,7 \\
\hline 6 & 66,8 & 13,3 & 13,3 & 1,6 & 10,0 \\
\hline 7 & 65,8 & 23,0 & 15,4 & 2,2 & 16,7 \\
\hline
\end{tabular}

ETR and ETM represent the quantities of water transpired in the presence of polyethylene glycol and in normally watered soil, respectively. These figures were obtained from plants cultivated under controlled conditions for one month. The other observations were taken on fieldgrown plants, at harvest. Root necrosis was assessed according to a scale from 0 (completely healthy roots) to 5 (roots entirely rotten).

TABLEAU 3

Relation entre la résistance à la sécheresse de quelques variétés et leur envahissement par M. phaseolina (résultats de 1983) et corrélation entre les divers paramètres mesurés.

Relation between drought tolerance and invasion by M. phaseolina (1983 results). Correlation between the parameters.

\begin{tabular}{|c|c|c|c|c|c|c|}
\hline $\begin{array}{c}\text { a) Observations } \\
\text { Variété }\end{array}$ & $\frac{\mathrm{ETR}}{\mathrm{ETM}} \times 100$ & $\begin{array}{l}\text { Efficience } \\
\text { de l'eau } \\
\text { (en } g / l \text { ) }\end{array}$ & $\begin{array}{c}\text { Intervalle } \\
\text { semis-floraison } \\
\text { (en j) }\end{array}$ & $\begin{array}{l}\text { Nombre de } \\
\text { plantes } \\
\text { sèches }\end{array}$ & $\begin{array}{c}\text { Nombre de } \\
\text { tiges contenant } \\
\text { des } \\
\text { microsclérotes }\end{array}$ & $\begin{array}{c}\text { Note moyenne } \\
\text { d'état sanitaire } \\
\text { des racines }\end{array}$ \\
\hline 12 & 72,8 & 3,12 & 91 & 8 & 6 & 4,1 \\
\hline 11 & 68,4 & 3,85 & 94 & 0 & 0 & 0,8 \\
\hline 26 & 64,5 & 3,71 & 95 & 4 & 3 & 3,4 \\
\hline 9 & 64,3 & 4,03 & 95 & 4 & 4 & 2,0 \\
\hline 1 & 63,4 & 3,99 & 96 & 7 & 5 & 2,5 \\
\hline 7 & 63,4 & 3,60 & 89 & 7 & 7 & 3,9 \\
\hline 38 & 61,1 & 3,41 & 92 & 4 & 3 & 2,0 \\
\hline 35 & 60,2 & 4,15 & 87 & 8 & 7 & 4,3 \\
\hline 22 & 59,4 & 3,81 & 86 & 7 & 6 & 4,0 \\
\hline 20 & 57,9 & 3,73 & 86 & 4 & 7 & 5,0 \\
\hline 18 & 57,9 & 4,62 & 90 & 7 & 7 & 3,9 \\
\hline 40 & 55,6 & 3,86 & 86 & 8 & 5 & 3,1 \\
\hline 31 & 55,0 & 3,79 & 94 & 8 & 4 & 2,5 \\
\hline 33 & 52,6 & 3,88 & 84 & 5 & 5 & 3,1 \\
\hline 23 & 52,1 & 3,87 & 91 & 6 & 6 & 3,9 \\
\hline 41 & 51,4 & 3,14 & 89 & 8 & 7 & 4,4 \\
\hline 34 & 50,0 & 3,60 & 93 & 8 & 5 & 3,1 \\
\hline 29 & 49,0 & 3,44 & 92 & 7 & 7 & 4,6 \\
\hline \multicolumn{7}{|l|}{ b) Corrélations } \\
\hline Efficience de l'eau & $-0,01$ & & & & & \\
\hline Intervalle semis-floraison & 0,30 & $-0,04$ & & & & \\
\hline Nombre de plantes sèches & $-0,38^{\circ}$ & $-0,11$ & $-0,24$ & & & \\
\hline $\begin{array}{l}\text { Tiges contenant des micro- } \\
\text { sclérotes }\end{array}$ & $-0,40^{\circ}$ & $-0,01$ & $-0,52^{*}$ & $0,72^{* *}$ & & \\
\hline Notation des racines & $-0,34$ & $-0,17$ & $-0,54^{*}$ & $0,56^{*}$ & $0,90^{* *}$ & \\
\hline
\end{tabular}

Les coefficients suivis des signes ${ }^{\circ},{ }^{*},{ }^{* *}$, sont significatifs aux seuils de 10,5 et 1 p. 100 , respectivement.

For ETR/ETM, see table 2. Water efficiency is the ratio between total dry weight after 4 weeks and the quantity of water consumed during the same length of time under controlled conditions. Coefficients followed by ${ }^{\circ},{ }^{*},{ }^{* *}$, were significant at 10,5 and $1 \%$ levels, respectively.

\section{Effet de la profondeur de semis}

Le taux de colonisation des racines par $M$. phaseolina demeure légèrement plus élevé, chez les plantes du semis profond, pendant toute la durée de la culture (fig. 3), mais les différences ne sont pas statistiquement significatives au seuil de 5 p. 100. La colonisation par les Fusarium spp. et les Cylindrocarpon spp. est plus forte dans le cas du semis profond, lors du 1er prélèvement ; ensuite les valeurs sont équivalentes.

En fin de culture, le nombre de plantes sèches est statistiquement le même dans les 3 traitements : de l'ordre de 11 p. 100. Cependant, la note qui correspond à l'état sanitaire des racines et la longueur moyenne des por- 


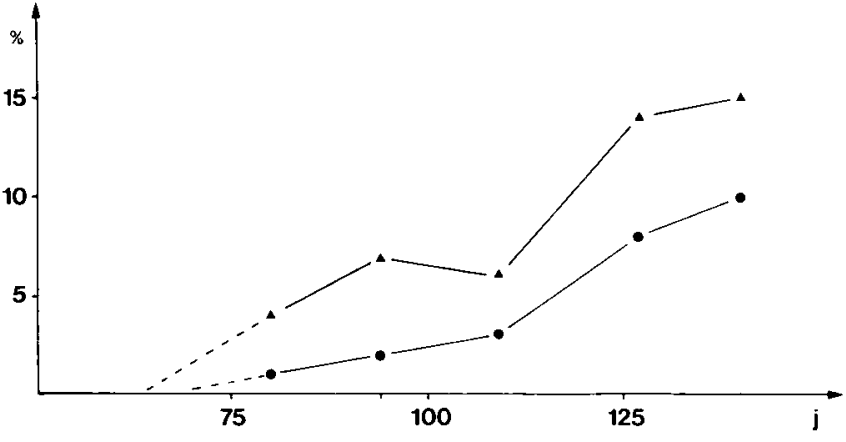

Figure 3

Evolution, en fonction du temps, du taux de colonisation par M. phaseolina des racines de tournesols semés le 16 avril, à $3 \mathrm{~cm}$ de profondeur $(\bullet)$ et à $7 \mathrm{~cm}$ de profondeur $(\boldsymbol{\Delta})$. Le temps est exprimé en nombre de jours après le semis.

Development with time of the percentage of colonization by M. phaseolina of roots of sunflowers seeded on 16 April, either at a depth of $3 \mathrm{~cm}(\bullet)$ or at a depth of $7 \mathrm{~cm}(\mathbf{\Delta})$. Time expressed as days from sowing date.

tions de moelle qui contiennent des microsclérotes sont significativement plus élevées chez les tournesols du semis profond (tabl. 4).

\section{E. Effet de la vigueur des plantes au début de la culture}

En 1983, la notation des tournesols au moment de la récolte fait apparaître une différence très nette entre les 2 catégories de plantes (tabl. 5). Quel que soit le critère envisagé, les végétaux qui ont eu une levée tardive ou un moins bon développement initial sont plus profondément affectés par les attaques parasitaires.

En 1984, c'est l'inverse que l'on constate : on observe moins de symptômes maladifs sur les plantes repérées comme chétives en début de culture (tabl. 6).

\section{DISCUSSION}

M. phaseolina est un champignon actif à des températures élevées, avec un optimum compris entre 28 et $35^{\circ} \mathrm{C}$ (Dhingra \& Sinclair, 1978) ; les isolements que nous avons étudiés n'échappent pas à cette règle (résultats non publiés). Il est bien certain que l'on est loin de cet optimum, en France, pendant la germination et le début du développement des tournesols. Il n'est donc pas très surprenant que le champignon ne se manifeste pas durant cette période. Cependant, le réchauffement du sol paraît bien être une condition nécessaire mais non suffisante pour la colonisation des tournesols par M. phaseolina. En effet, dans notre essai de semis tardif, en juin, la température moyenne du sol oscille entre 21 et $24^{\circ} \mathrm{C}$ à partir. du $30^{\mathrm{e}} \mathrm{j}$ après le semis. Mais $c^{\prime}$ 'est seulement vers le $75^{\mathrm{e}} \mathrm{j}$ que $M$. phaseolina peut être isolé des plantes : ce moment n'est pas marqué par une élévation de la température (au contraire, elle commence à décroître) mais par le début de la floraison des tournesols (fig. 2). D'autre part, dans un autre essai (tabl. 1), les tiges des tournesols privés de capitules ne sont pas envahies, alors que les plantes témoins voisines
TABLEAU 4

Effets de la profondeur du semis.

Effects of sowing depth on charcoal-rot symptoms.

\begin{tabular}{lccc}
\hline \hline & $\begin{array}{c}\text { Pourcentage } \\
\text { de plantes } \\
\text { sèches }\end{array}$ & $\begin{array}{c}\text { Longueur } \\
\text { moyenne des } \\
\text { lésions dans } \\
\text { la moelle } \\
(\text { en cm) }\end{array}$ & $\begin{array}{c}\text { Note } \\
\text { moyenne } \\
\text { d'état } \\
\text { sanitaire } \\
\text { des racines }\end{array}$ \\
\hline $\begin{array}{c}\text { Semis normal } \\
(3-4 \mathrm{~cm})\end{array}$ & $8,3 \mathrm{a}$ & $3,96 \mathrm{a}$ & $2,86 \mathrm{a}$ \\
$\begin{array}{c}\text { Semis moyen } \\
(5-6 \mathrm{~cm})\end{array}$ & $11,1 \mathrm{a}$ & $3,60 \mathrm{a}$ & $2,94 \mathrm{ab}$ \\
$\begin{array}{c}\text { Semis profond } \\
(7-8 \mathrm{~cm})\end{array}$ & $13,9 \mathrm{a}$ & $6,93 \mathrm{~b}$ & $3,03 \mathrm{~b}$ \\
\hline \hline
\end{tabular}

Les chiffres suivis de lettres différentes sont significativement différents au seuil de 5 p. 100 (tests de $X^{2}$ et de $t$ ).

Figures followed by different letters were significantly different at $5 \%$ level ( $\mathrm{X}^{2}$ and Student's $\mathrm{t}$ tests).

\section{TABLEAU 5}

Relation entre la vigueur initiale des plantes et l'envahissement par M. phaseolina (essai de 1983).

Charcoal rot symptoms observed on 7 September 1983, on sunflowers scored on 13 May as : a) weak; b) normal.

\begin{tabular}{lccc}
\hline \hline & $\begin{array}{c}\text { Pourcentage } \\
\text { de plantes } \\
\text { sèches }\end{array}$ & $\begin{array}{c}\text { Pourcentage } \\
\text { de tiges } \\
\text { contenant } \\
\text { des } \\
\text { microsclérotes }\end{array}$ & $\begin{array}{c}\text { Note } \\
\text { moyenne } \\
\text { d'état } \\
\text { sanitaire } \\
\text { des racines }\end{array}$ \\
\hline $\begin{array}{l}\text { a) Plantes faibles en } \\
\text { début de végétation }\end{array}$ & 68,1 & 60,6 & 3,80 \\
b) Plantes « normales" & 36,4 & 42,4 & 2,69 \\
\hline
\end{tabular}

Pour les deux premiers critères, les pourcentages indiqués sont différents au seuil de 1 p. 100 selon le test de $X^{2}$. Les notes racinaires sont différentes au seuil de 5 p. 100 selon le test de $t$.

Percentages of dry plants and percentages of stalks with microsclerotia were different at $1 \%$ level $\left(\mathrm{X}^{2}\right.$ test). Estimates of root necrosis were different at $5 \%$ level (Student's t test).

\section{TABLEAU 6}

Relation entre la vigueur initiale des plantes et l'envahissement par M. phaseolina (résultats de 1984).

Charcoal-rot symptoms observed on 4 September 1984, on sunflowers which had been noted on 15 May, as : a) weak; b) normal.

\begin{tabular}{|c|c|c|c|}
\hline & $\begin{array}{l}\text { Pourcentage } \\
\text { de plantes } \\
\text { sèches }\end{array}$ & $\begin{array}{l}\text { Longueur } \\
\text { moyenne des } \\
\text { lésions dans } \\
\text { la moelle } \\
(\mathrm{en} \mathrm{cm})\end{array}$ & $\begin{array}{c}\text { Note } \\
\text { moyenne } \\
\text { d'état } \\
\text { sanitaire } \\
\text { des racines }\end{array}$ \\
\hline $\begin{array}{l}\text { a) Plantes faibles en } \\
\text { début de végétation }\end{array}$ & 13,9 & 1,14 & 2,53 \\
\hline b) Plantes «normales » & 34,0 & 7,15 & 3,14 \\
\hline
\end{tabular}

Pour chaque critère, les valeurs indiquées sont significativement différentes au seuil de 1 p. 100 (test de $\mathrm{X}^{2}$ et test de $t$, respectivement).

For each series of observations, figures were different at $1 \%$ level ( $\mathrm{X}^{2}$ and Student's t test, respectively). 
sont colonisées, ce qui montre que la température n'est pas, dans ce cas, un facteur limitant.

Le rôle déterminant du déclenchement des phénomènes reproductifs dans la sensibilisation des tournesols à $M$. phaseolina est bien mis en évidence par cette expérience de décapitation des tiges : lorsque les fleurs sont supprimées, le développement végétatif se poursuit et les racines et les tiges demeurent saines. WYLLIE \& CALVERT (1969) ont réalisé une expérience du même genre sur le soja : lorsqu'ils suppriment les fleurs au fur et à mesure de leur apparition, ils n'observent plus la formation d'aucun sclérote dans les tiges, alors même que le champignon est présent. Ils en déduisent que l'apparition des microsclérotes est corrélée avec la formation des gousses. Leurs données ne permettent malheureusement pas de faire de comparaison entre l'aspect général des plantes dans chacun de leurs traitements. Dans notre essai, les racines des plantes décapitées, bien que saines d'aspect, hébergent autant de $M$. phaseolina que les autres à la fin de l'été. On peut considérer que le champignon se trouve en infection latente dans les racines, comme il l'est probablement dans les tissus des sojas de WYLLIE \& CALVERT. Mais la moelle des tiges des tournesols n'est envahie qu'après la floraison (SAUMON et al., 1984).

C'est donc, au-dessus d'un seuil de température minimum, le stade de développement des tournesols qui semble déterminer le moment de l'invasion des tiges et de l'extériorisation des symptômes. La rapidité et l'intensité de cet envahissement peuvent cependant être modulées par divers facteurs extérieurs : nous en avons étudié quelques-uns au cours de ce travail. Tous ces facteurs ont un caractère commun : ils contribuent à affaiblir les plantes.

Ainsi, l'ablation d'une partie des feuilles diminue l'activité photosynthétique. Les conséquences de cette opération sont d'autant plus nettes que les feuilles supprimées sont plus jeunes, donc plus actives. Nous constatons en effet que la perte des étages supérieurs est plus grave que celle des feuilles basses (tabl. 1). Un tel problème peut se rencontrer, dans la pratique, après l'attaque de ravageurs ou de parasites foliaires. La carence en bore, mise en évidence dans plusieurs départements du sud de la France, provoque aussi une " grillure » des feuilles et il a été observé, à l'occasion de plusieurs inventaires, que les plantes «grillées» étaient davantage attaquées par $M$. phaseolina (PERNY, comm. pers.). Il est d'ailleurs possible que cette sensibilisation provoquée par la carence en bore ait pu, dans le passé, faire attribuer à tort le syndrome de « grillure " à $M$. phaseolina.

Il nous a été donné d'observer des tournesols semés sur un ancien verger de pêchers, habituellement désherbé au bromacil. Les plantes avaient une croissance normale à la place naguère occupée par les arbres mais, entre les arbres, des résidus d'herbicide avaient entraîné de sérieux retards de développement. Dans ces zones polluées, 57 p. 100 des tiges contenaient des microsclérotes en fin de culture, au lieu de 40 p. 100 seulement dans les parties non traitées.

El MAHjouB et al. (1979) ont montré, de leur côté, que l'arrosage des tournesols avec des eaux chargées en sel les sensibilise au dessèchement précoce. L'importance des attaques de $M$. phaseolina dans les tiges varie dans le même sens que la concentration en $\mathrm{NaCl}$ des eaux d'irrigation.

La sécheresse est un facteur de sensibilisation à M. phaseolina connu depuis longtemps. Ses effets ont été démontrés dans le cas des pépinières de pins (HODGES, 1962), du sorgho (EDMUNDS, 1964), du cotonnier (GHAFFAR \& ERWIN, 1969), du haricot (MAGAlHÃES et al., 1982) et, plus récemment, du tournesol (BLANCO-LÓPEZ \& JiMÉNEZ-DíAZ, 1983). Toutes choses égales par ailleurs, le pourcentage de plantes malades est toujours plus faible lorsque les cultures sont irriguées ou situées dans des sols profonds et frais (SAUMON et al., 1984). Une variété sélectionnée pour sa résistance à la sécheresse aura-t-elle donc un meilleur comportement que les autres vis-à-vis de $M$. phaseolina? Nos essais de comportement variétal apportent quelques éléments de réponse à cette question. Si l'on prend comme critère de résistance à la sécheresse le rapport ETR/ETM, on peut dire que la résistance à la maladie varie dans le même sens que ce paramètre. Cette constatation doit cependant être nuancée. Les hybrides mis en comparaison ont en effet des degrés de précocité différents, alors que les dates des semis et des relevés sont les mêmes pour tous. De ce fait les variétés les plus précoces, ayant été envahies les premières, se trouvent pénalisées, ce qui explique sans doute la bonne corrélation négative observée entre l'intervalle semis-floraison et les paramètres caractérisant l'état sanitaire des plantes (tabl. 3). La corrélation avec l'efficience de l'eau n'est pas bonne : ce paramètre prend en compte la production de matière sèche, alors que le rapport $\mathrm{ETR} / \mathrm{ETM}$ reflète plus directement la possibilité pour la plante d'extraire de l'eau dans des conditions défavorables.

Si la sécheresse est la principale cause d'affaiblissement des plantes, d'autres facteurs de sensibilisation peuvent encore être envisagés. Nous avons montré qu'un semis trop profond, entraînant une levée difficile, favorise $M$. phaseolina. Ainsi, un retard de végétation enregistré dès le début de la culture peut sensibiliser les tournesols à une invasion qui n'aura lieu que 2 mois plus tard. Ceci est confirmé par les résultats de l'essai d'indexation de 1983 (tabl. 5). Les résultats apparemment contradictoires de 1984 pourraient s'expliquer par la pluviométrie particulière de cette année-là (fig. 1). Le mois de mai 1984 a été en effet exceptionnellement pluvieux, assurant de bonnes réserves en eau jusqu'à la floraison. Les tournesols ont donc fleuri dans de bonnes conditions, les plus vigoureux portant un nombre d'akènes supérieur à la moyenne. Il est vraisemblable que la forte demande de tels capitules pendant le remplissage des graines, moment où la photosynthèse s'est trouvée réduite par le manque d'eau, a entraîné un déséquilibre nutritionnel, une perturbation des relations source-puits, se traduisant par une sénescence précoce.

Notre conception du dessèchement précoce des tournesols est ainsi très proche des hypothèses de DODD (1980) et de BARRIÈrE et al. (1981) sur la pourriture des tiges de maïs. Selon DoDD, le remplissage des grains sur les épis provoque un épuisement des réserves de sucres accumulées dans les tiges et les racines. Cet appauvrissement entraîne la sénescence et l'affaiblissement des mécanismes de défense du maïs et l'invasion des racines par divers parasites peu spé- 
cialisés, qui gagnent ensuite les tiges. BARRIÈRE \& GAY (1984), et plus récemment BARRIÈRE (1985), ont bien mis en évidence la relation entre les carences nutritionnelles entraînées par les déséquilibres sourcepuits et l'initiation de la sénescence des organes du maïs.

Chez le tournesol, l'invasion des tiges par $M$. phaseolina (et aussi par d'autres parasites de faiblesse) nous paraît également liée à l'apparition de cette sénescence. Ce phénomène, qui constitue une étape normale du développement des plantes, génétiquement déterminée, peut être accéléré et intensifié par tous les facteurs susceptibles d'intervenir sur la physiologie des plantes. Il existe d'ailleurs une relation entre l'âge des plantes, la teneur en sucres de la base des tiges et l'invasion parasitaire de ces organes, chez le tournesol (DAVET \& SERIEYS, 1986).

Reçu le 13 février 1986. Accepté le 6 juin 1986.

\section{REMERCIEMENTS}

Ce travail a été réalisé en collaboration avec le Centre Technique Interprofessionnel des Oléagineux Métropolitains et grâce à son concours financier. Nous remercions M. MAILHOL pour l'aide qu'il nous a apportée pour la mise en place des essais sur les terrains de la Coopérative Agricole Lauragaise.

\section{RÉFÉRENCES BIBLIOGRAPHIQUES}

Acimović M., 1962. Sclerotium bataticola Taub. as a causal agent of sunflower wilt in Vojvodina. Zast. Bilja, 69-70, 125-138.

Alabouvette C., Bremeersch P., 1975. Les dégâts provoqués par Macrophomina phaseoli dans les cultures de tournesol. Déf. Vég., 173, 125-131.

Alissa A. A., 1982. Etude de la croissance du tournesol thelianthus annuus $L$.) sous contrainte hydrique. Recherche d'un test de sélection pour la résistance à la sécheresse. Mémoire de DEA, E. N.S.A. Montpellier, $38 \mathrm{p}$.

Barrière Y., 1985. Aspects physiologiques de la pourriture des tiges du maïs. Agronomie, 5, 361-368.

Barrière Y., Gay J. P., 1984. Approche physiologique de la sénescence du maïs, 355-365. In Gal.t.Als A. : "Physiologie du maïs ». I.N.R.A., $574 \mathrm{p}$.

Barrière Y., Panouillé A., Cassini R., 1981. Relations source-puits et sélection du maïs pour la résistance à la pourriture des tiges. Agronomie, 1, 707-711.

Barros M. L., 1980. Informação sobre as doenças do girassol em Portugal. Garcia de Orta, Série Est. Agron., 7, 21-28.

Blanchet R., Cavalié G., Cruiziat P., Morizet J., Durrieu G., Merrien A., Planchon C., Ribeyre C., Manichon H., 1983. Physiologie de la formation du rendement chez le tournesol. Inform. Tech. C.E.T.I.O.M., 83, 72 p.

Blanco-López M. A., Jiménez-Díaz R. M., 1983. Effect of irrigation on susceptibility of sunflower to Macrophomina phaseoli. Plant Dis., 67, 1214-1217.

Davet P., Serieys H., 1986. Relation entre la teneur en sucres réducteurs des tissus du tournesol (Helianthus annuus L.) et leur invasion par Macrophomina phaseolina (Tassi) Goid. Phytopathol. Z., 115, sous presse.

Dhingra O. D., Sinclair J. B., 1978. Biology and pathology of Macrophomina phaseolina. Univ. Federal Viçosa, Minas Gerais, Brésil, 166 p.
Dodd J. L., 1980. Grain sink size and predisposition of Zea mays to stalk rot. Phytopathology, 70, 534-535.

Edmunds L. K., 1964. Combined relation of plant maturity, temperature, and soil moisture to charcoal stalk rot development in grain sorghum. Phytopathology, 54, 514-517.

El Mahjoub M., Bouzaidi A., Jouhri A., Hamrouni A., El Beji, 1979. Influence de la salinité des eaux d'irrigation sur la sensibilité du tournesol au Macrophomina phaseoli (Maubl.) Ashby. Ann. Phytopathol., 11, 61-67.

Ghaffar A., Erwin D. C., 1969. Effect of soil water stress on root rot of cotton caused by Macrophomina phaseoli. Phytopathology, 59, 795-797.

Hodges C. S., 1962. Black root rot of pine seedlings. Phytopatho$\log y, 52,210-219$.

Mc Cain A. H., Smith R. S., 1972. Quantitative assay of Macrophomina phaseoli from soil. Phytopathology, 62, 1098.

Magalhães de A. A., Choudhury M. M., Millar A. A., De Albuquerque M. M., 1982. Efeito do déficit de água no solo sobre o ataque de Macrophomina phaseolina em feijão. Pesqui. agropecu. brasi., Ser. Agron., 17, 407-411.

Messiaen C. M., Lafon R., 1965. Les maladies des plantes maraîchères, vol. II, I.N.R.A., Paris, 331 p.

Sackston W. E.. Jiménez-Díaz R. M., 1976. Diseases of sunflower in Spain. Proc. Int. Sunflower Conf., 7th., vol. 2, 194-199.

Saumon E., Herbach M., Goore B. K., Davet P., 1984. Le dessèchement précoce des tournesols. Dynamique de la colonisation des plantes par les champignons du sol et envahissement tardif par Macrophomina phaseolina. Agronomie, 4, 805-812.

Wyllie T. D., Calvert O. H., 1969. Effect of flower removal and pod set on formation of sclerotia and infection of Glycine max by Macrophomina phaseoli. Phytopathology, 59, 1243-1245. 